\title{
Impact of Selectively Implementing Control Flow Error Detection Techniques
}

\author{
Jens Vankeirsbilck*, Jonas Van Waes, Hans Hallez, Jeroen Boydens \\ Department of Computer Science, KU Leuven Bruges Campus, Spoorwegstraat 12, 8200 Brugge, Belgium
}

\begin{abstract}
Many software-implemented control flow error detection techniques have been proposed over the years. In an effort to reduce their overhead, recent research has focused on selective approaches. However, correctly applying these approaches can be difficult because their respective literature gives little guidance on the practical implementation. This paper aims to address this concern and proposes a new approach. Our new approach is easier to implement and is applicable on any existing control flow error detection technique. To prove its validity, we apply our new approach to five different control flow error detection techniques and perform fault injection experiments. The results show that the selective implementation, while decreasing the imposed overhead, can have a negative impact on error detection ratio.
\end{abstract}

Keywords: Control Flow Error, Software-Implemented Error Detection, Selective Implementation

\section{Introduction}

The reliability of embedded systems in ever harsher working environments is becoming more important. These systems are, however, more vulnerable to external disturbances which introduce bit-flips in the system's hardware. Research shows that particles, such as alpha particles, neutrons and muons, have the energy to strike microcontroller components, such as internal memory cells, and change their state $[1,2,3,4,5]$. Different sources from literature show that the soft error rate of different devices increases for each new technology generation $[2,3,4]$. Next to particles, a second source of bit-flips is electromagnetic interference (EMI), which accumulates charges on PCB traces, on transistors, etc., and changes their state [6, 7, 8]. EMI has an ever more negative impact on reliability, as more systems are being used close to one another and wireless communication is being increasingly used. Jagannathan et al. demonstrate that the sensitivity of transistors and memory cells increases with temperature. They show that a higher microcontroller temperature increases the effect generated by a striking particle or by EMI and decreases the drive strength

\footnotetext{
* Corresponding author

Email addresses: jens.vankeirsbilck@kuleuven.be (Jens Vankeirsbilck), jonas.vanwaes@kuleuven.be (Jonas Van Waes), hans.hallez@kuleuven.be (Hans Hallez), jeroen.boydens@kuleuven.be (Jeroen Boydens) 
of transistors used in the components [9]. And, although not the scope of this paper, research from the security domain also shows that external attackers can introduce bit-flips in order to extract critical data, such as PIN codes of smart cards and cryptographic keys [10, 11]. The combination of these sources has increased the number of erroneous bit-flips to more than one per day for each device and thus makes them a real concern for all embedded system engineers [12].

These introduced bit-flips can cause invalid behavior such as erroneously controlling an actuator, corrupting data or corrupting the execution order of instructions. The corruption of the execution order of instructions is also known as a control flow error (CFE). A CFE is a violation against the control flow graph (CFG) of the program. The CFG is a representation of the program, in which the program is divided into basic blocks and edges. A basic block is a sequence of consecutive instructions with exactly one entry and one exit point. An edge is an intentional path between basic blocks. CFEs are typically partitioned into two categories: inter-block CFEs and intra-block CFEs. An inter-block CFE is an invalid jump through the program between two different basic blocks, while an intra-block CFE is an invalid jump within the same basic block. Both types of CFEs can cause the affected program or system to halt, to crash or to provide erroneous output, potentially leading to hazardous situations.

To increase the reliability of embedded systems, several software-implemented CFE detection techniques, have been proposed $[13,14]$. In a previous comparative study, we developed a CFE detection technique called RACFED which has a higher detection ratio at a lower execution time overhead when compared to similar techniques [15]. The imposed execution time overhead, however, is still relatively high. Therefore, research has focused on selective CFE detection techniques $[16,17]$. Selective CFE detection techniques only protect a selected part of the target program to reduce the imposed execution time overhead. Whilst reducing the execution time overhead, selective techniques are often difficult to implement correctly or are difficult to apply to other CFE detection techniques.

This paper aims to address this concern by proposing a new principle to selectively implement CFE detection techniques. Our new principle is relatively easy to implement and can be used on any CFE detection technique. To validate this principle, we apply it to five CFE detection techniques: our RACFED technique, the RSCFC technique, the SIED technique, the CFCSS technique and the YACCA_CMP technique $[13,14,15,18,19]$. The rest of this paper is organized as follows. We discuss more background and related work in Section 2. Next, we discuss how to create the selective versions of the five selected techniques in Section 3. Then, we validate these selective versions and present the experiment setup and the results in Section 4 and Section 5, respectively. Finally, future work is presented in Section 6 and conclusions are drawn in Section 7. 


\section{Background and Related Work}

Past years, a multitude of CFE detection techniques has been proposed [13, 14, 18, 19, 20, 21, 22]. In order to detect CFEs, such techniques insert extra control variables, called signatures, and validation instructions into the target program at compile time. At run time, the added instructions recalculate these control variables and compare them to their expected compile-time value. A mismatch indicates that a CFE has been detected. In previous work, the authors performed a comparative study of these techniques and used the data to develop a new CFE detection technique called Random Additive Control Flow Error Detection (RACFED) [15]. When compared to similar techniques, RACFED shows to be the better technique since it detects more CFEs at a lower overhead.

The above mentioned techniques are full implementation techniques, i.e., they insert the control variable update and verification instructions in each basic block in the CFG. In recent years, selective implementations have been proposed, where the extra instructions are only inserted in certain selected basic blocks. One way to selectively apply CFE detection, is to simply ignore some basic blocks. This approach is taken by the S-SETA technique [16]. Within S-SETA, smaller basic blocks have no extra instructions inserted in order to reduce the imposed execution time overhead. While this drastically reduces the overhead, it also reduces the detection ratio, as CFEs that occur within the ignored basic blocks are potentially undetectable.

A second way to to selectively apply CFE detection, is to insert signature update instructions in each basic block, but to only insert signature verification instructions in selected basic blocks. Chielle et al. propose to only insert the verification instructions in larger basic blocks with their SETA-C technique [16]. Khudia et al. took another approach with their Abstract Control Signatures (ACS) technique [17]. They divide the basic blocks into regions and assign a specific signature to each region. Each basic block in a region has a signature update instruction inserted, while signature verification instructions are only inserted in basic blocks where the control flow changes from one region to another. Khudia et al. define their regions as a collection of basic blocks which has a single entry point but multiple exit points. Ideally, the regions should possess certain properties that help in minimizing the number of signature updates and verifications.

Although SETA-C and ACS show promise, implementing them can be difficult. SETA-C only inserts the verification instructions in larger basic blocks. For algorithms with little difference between the length in basic blocks, the decrease in execution time overhead can be limited. To gain the maximum decrease in execution time overhead when applying ACS, the division of the CFG into regions is critical. However, Khudia et al. provide little guidance on how to find this optimal division.

Therefore, we propose an easier and more consistent approach to selectively implement CFE detection techniques. We propose to only insert the signature verification instructions in return basic blocks. These are basic blocks which contain the return statement that exits out of the current function or program and returns control to the calling function or program. These basic blocks are the last basic block in each possible 
path through the target CFG and thus allow to detect all CFEs that occur within the CFG.

\section{Selective Implementation}

To demonstrate that our approach is indeed easy to use on different CFE detection techniques, we applied the principle to five selected CFE detection techniques: RACFED, RSCFC, SIED, CFCSS and YACCA_CMP. We selected our RACFED technique because previous work shows it outperforms similar techniques, i.e. RSCFC and SIED. To prove that our proposed selective implementation strategy not only works for techniques that detect both inter-block and intra-block CFEs, we selected the CFCSS and YACCA_CMP techniques which only detect inter-block CFEs. First, we discuss how the principle transforms a technique into its selective version with a high-level example. For the remainder of this paper, the selective version of a technique is indicates as S-technique. Next, we present the small change to be made to the compile-time process to implement each technique selectively. This section will use our RACFED technique as running example, but the principles stand for all five techniques.

\subsection{High-level example}

RACFED is our CFE detection technique that uses run-time signature updates to detect both inter- and intra-block CFEs. A high-level implementation of RACFED is shown on the left-hand side of Fig. 1. The instructions inserted by RACFED are indicated in bold and $S$ is the run-time signature. As can be seen, a signature update is inserted at the beginning of each basic block and after each original non-jump instruction. In RACFED, each basic block has a signature verification instruction that compares the run-time signature with the compile-time value. These are indicated as $\mathrm{S} !=$ \#value: error(). When a mismatch between the run-time and compile-time value is detected, a call is made to the error-handler.

Applying our proposed selective implementation approach transforms RACFED in S-RACFED. As shown in the right-hand side of Fig 1, only the bottom basic block has a signature verification instruction inserted because that is the return basic block. Since S-RACFED does not remove any signature update instructions, it should have the same CFE detection ratio as RACFED but with a lower execution time overhead.

\subsection{Change in Compile-time Process}

There are four steps in the compile-time process to implement RACFED:

1. First, all needed compile-time variables are assigned to all basic blocks.

2. Then, the instruction monitoring is implemented. These update instructions help to detect intra-block CFEs.

3. Next, the first signature update and the signature verification instructions are inserted in each basic block. 


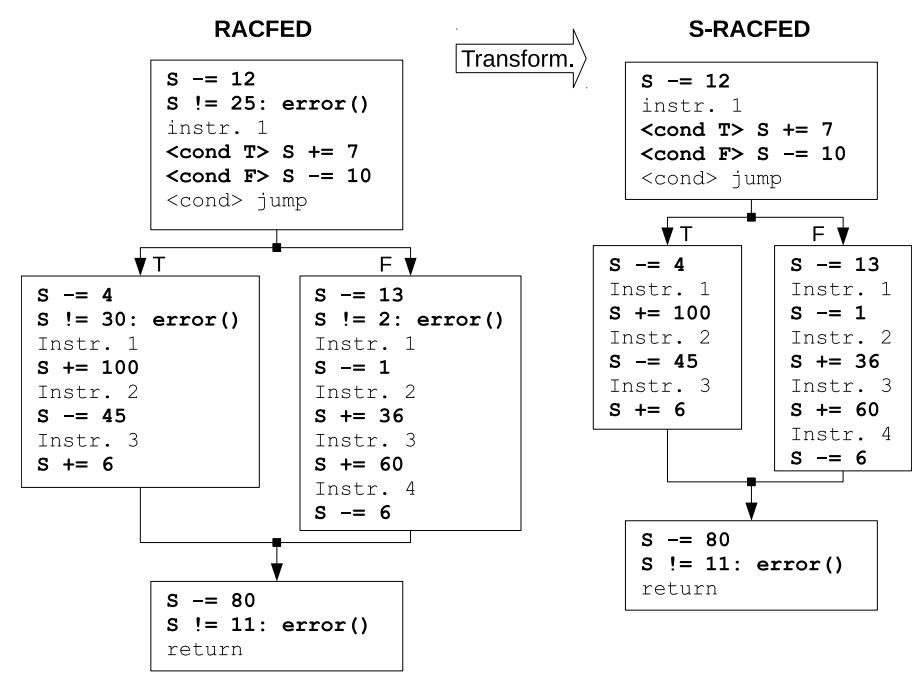

Figure 1: Transformation from RACFED to S-RACFED.

4. Finally, the last signature update is inserted in each basic block.

To implement S-RACFED, the third step is slightly adjusted. The first signature update instruction is still inserted in each basic block. The signature verification, however, is only inserted in return basic blocks. Therefore, an extra check is inserted in the compile-time process that analyses whether or not the last instruction of the basic block is a return instruction. If it is, only then the signature verification is inserted in the basic block.

\section{Experimental Setup}

In order to thoroughly validate the proposed approach, we performed fault injection experiments, measured the error detection cost and measured the error detection latency of both the full and the selective implementations. This section discusses the setup of the performed experiments in detail. First, we present the four criteria we measured. Next, we present the selected case studies and the selected target. Finally, we discuss the used fault injection process.

\subsection{Criteria}

To compare the selective versions with the full versions of each technique, we measured four criteria: the effect of each error, the execution time overhead, the code size overhead and the error detection latency.

\subsubsection{Error Effects}

For every injected fault, we determined its effect. We grouped the effects in four categories: 
- Detected (Det.): This percentage of faults was detected by the implemented countermeasure. In other words, this category is the desired result.

- Hardware Detection (HD): Many processors already have several internal fault handlers that are able to detect specific hardware faults, such as improper bus usage or memory access violations. This category represents the faults detected by such fault handler.

- Silent Data Corruption (SDC): These are the faults that were not detected by the implemented technique and caused the algorithm to produce a wrong result.

- No Effect (NE): Finally, this is the percentage of the faults that were not detected and did not affect the outcome of the target algorithm.

\subsubsection{Error Detection Cost}

Software-implemented CFE detection techniques insert extra instructions into the code they need to protect. This means that the code size and the execution time of the protected code is higher than that of the unprotected code. We measured both its code size and execution time and compared the results to the unprotected code, as shown by eq. (1) and eq. (2). The code size is measured by counting the number of instructions in the code. The execution time is measured using a hardware timer of the target.

$$
\text { code size relative to unprot. } \text { code }=\frac{\text { instruction count protected }}{\text { instruction count unprotected }}
$$

exec. time relative to unprot. code $=\frac{\text { exec. time protected }}{\text { exec. time unprotected }}$

\subsubsection{Error Detection Latency}

Because the main difference between the full implementation and the selective implementation is the placement of the verification instruction, we measured the error detection latency of both versions. For every detected CFE, we counted the number of executed instructions before the CFE was detected.

\subsection{Case Studies}

The selected case studies are an implementation of the following eight algorithms:

- Bit Count (BC): The bit count algorithm counts the bits set, i.e. the 1 's, in the given data word, also known as the hamming weight. This functionality is, amongst others, used in the communication domain to calculate a parity bit or in the cryptographic domain to calculate keys [23].

- Bubble Sort (BS) and Quicksort (QS): The bubble sort and quick sort algorithms were selected because the sorting of information is used in different systems, e.g., to assign priorities or to enable faster analysis of data [24]. 
- Cyclic Redundancy Check (CRC): The cyclic redundancy check algorithm is mainly used to add error detection information in data transmissions [25]. When using CRC, additional bits are added to the transmitted data which enables to analyze whether or not the received information is correct.

- Cubic Solver (CU): The cubic function solver algorithm is used in physics related applications, such as calculating the speed of a car or calculating the power density in wind turbines [26].

- Dijkstra's algorithm (DIJ): Dijkstra's algorithm calculates the shortest path between different nodes, which is a highly used functionality in routing applications [27].

- Fast Fourier Transform (FFT): The fast fourier transform is used in a wide variety of applications, such as file compression, voice recognition and vibro-acoustic analysis in cars [28].

- Matrix Multiplication (MM): The matrix multiplication algorithm was selected because matrices and matrix multiplication are used in many different embedded domains such as image processing, e.g., CAT and MRI scan, robotics and data compression [29].

While the BS, MM, and QS case studies use our implementation, the implementation of the remaining case studies was selected from MiBench version 1.0 [30]. MiBench is a free commercially representative embedded benchmark suite. This suite contains 35 embedded applications for benchmarking purposes which are divided into six categories: Automotive and Industrial Control, Consumer Devices, Office Automation, Networking, Security and Telecommunications.

With the selected case studies, a wide array of embedded application domains is covered. The BS, QS, MM and FFT case studies are also the typical applications used to validate error detection techniques in literature $[13,14,18,19,20,21,22]$. Furthermore, they also have varying basic block and edge distributions which assures a thorough validation of our approach. Some setup and validation code is required to launch the case studies and verify their results. However, only the function implementing the target algorithm will have (S-)RACFED applied and only that function will be subjected to CFEs.

\subsection{Automatic Implementation}

To automatically implement the techniques in the low-level code of the selected case studies, we used our GCC plugin $^{1}$ [31]. This in-house developed plugin automatically adds the selected CFE detection technique to the low-level code of the target program. Currently, it supports up to ten full implementation CFE detection techniques and two ARM instructions architecture sets, ARMv6-M and ARMv7-M. To enable selective implementation, we not only applied the change to the implementation process of RACFED as

\footnotetext{
${ }^{1}$ The GCC plugin is available as open-source project on Github, licensed under the MIT Expat license: https://github. com/MGroupKULeuvenBrugesCampus/CFED_Plugin
} 


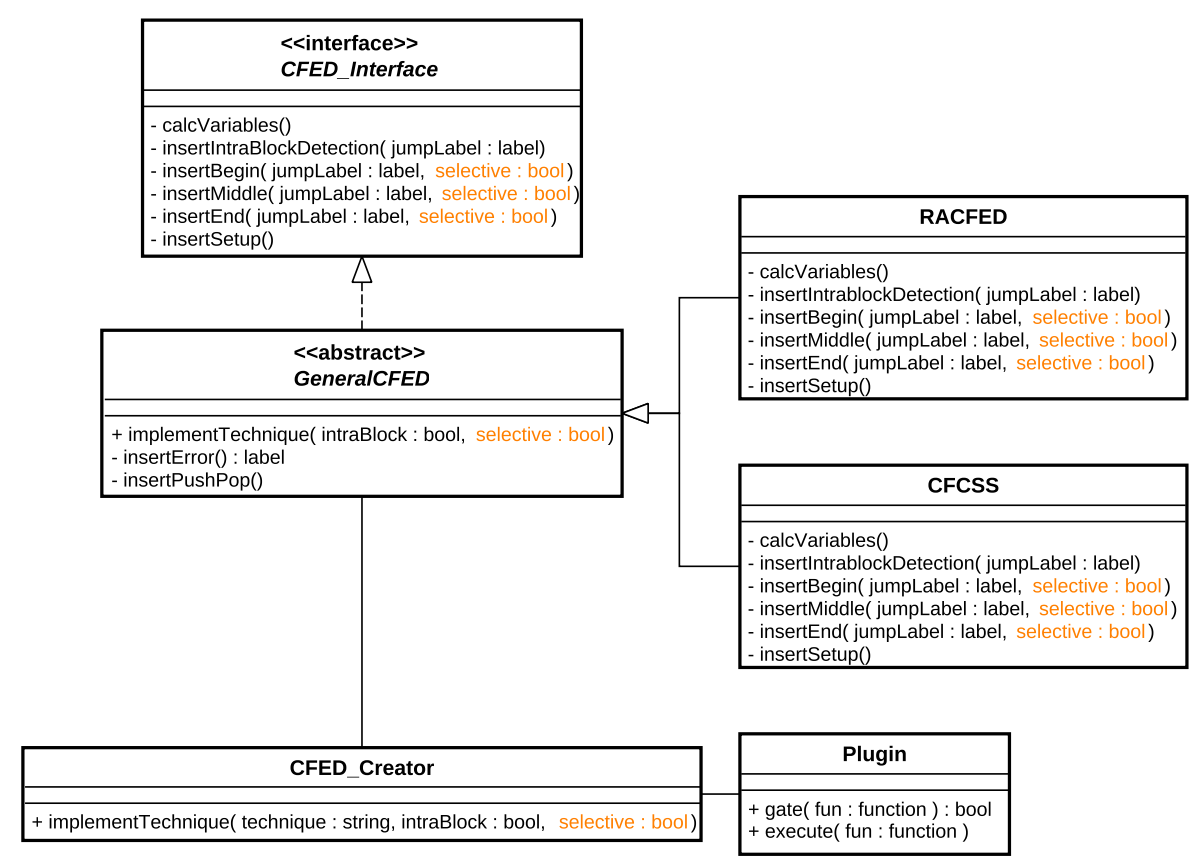

Figure 2: UML class diagram showing the implementation of our GCC plugin in pseudo-code, with the changes to enable selective implementation indicated in orange.

described in Section 3.2, the internal working of the plugin itself was also expanded as shown in orange on Fig. 2.

As shown in Listing 1, to use the plugin and implement a technique the compiler flags of the target program has to be adjusted. In total, seven extra compiler flags have to be added, i.e. five plugin-related flags and two global compilation flags. The global compilation flags, -ffixed-r11 and -ffixed-r6, tell the compiler not to use registers $r 11$ and $r 6$ during compilation. The plugin will use the first register to implement the chosen technique and the latter as stack pointer for the run-time variable stack. The five plugin-related flags specify where to find the plugin (line 4), which function to protect (line 6), what type of technique to apply (line 8), which technique to implement (line 10) and whether or not to implement it selectively (line 12). In our example, the flags have been set to only protect the $\mathrm{BC}$ algorithm itself, i.e. function=bit_count, and to implement the S-RACFED technique with both intra-block and interblock CFE detection, i.e. techniqueType=fullCFED, technique=RACFED, selective=1. For a better understanding of the different plugin-related flags and more explanation on the internal working of our GCC plugin, the reader is referred to [31]. 


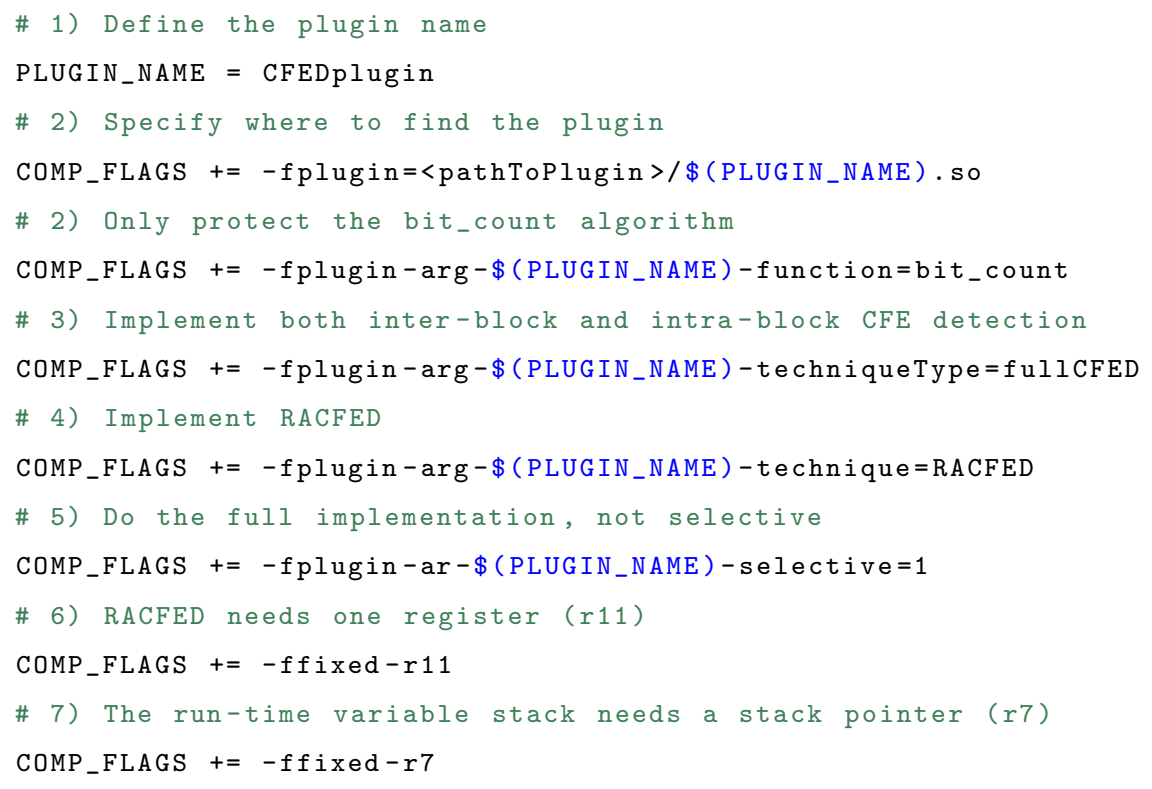

Listing 1: Adjustment to be made to the compiler flags to use our plugin

\subsection{Target}

We executed the case studies on an ARM Cortex-M3, because it is an industry-leading 32-bit processor used in many different embedded application domains.

For the CFE injection experiments, we used a simulated Cortex-M3 provided by the Imperas simulator [32]. The Imperas simulator is an instruction set simulator which allows to execute the target instructions at host (computer) speed. The Imperas simulator supports ARM, MIPS, PowerPC and several other targets. We used a simulated target for the fault injection because previous experiments showed that using a physical target suffers from communication overhead and communication errors. The communication overhead arises from using either USB or JTAG to communicate with the target, while the errors occur due to failure of the on-chip debugger that is used to modify the CPU registers. By switching to a simulated target, these disadvantages were eliminated.

Because the Imperas simulator is an instruction accurate simulator and not a cycle accurate simulator, it is less suited to perform timing measurements. Therefore, we performed the execution time measurements on a physical target, the NXP LPC 1768. The NXP LPC 1768 is an ARM Cortex-M3 driven microcontroller running at $96 \mathrm{MHz}$, including $512 \mathrm{kB}$ FLASH and $32 \mathrm{kB}$ RAM.

\subsection{Fault Injection Process}

The used CFE injection process is shown in Fig. 3 and starts with an initialization phase that initializes variables and creates all possible CFEs. Those possible CFEs are constructed from the user-defined options 
disasmFile and range. The disasmFile holds the path to the disassembly file of the target program. This file is needed to know which program counter $(\mathrm{PC})$ values are valid for the program. The range option indicates for which PC range the target program will be tested. The option allows to target a specific function (or functions) to be tested, instead of the entire program. All created possible CFEs are within this range.

Once the possible CFEs are created, the execution part of the fault injection process starts. This phase begins with performing a check to determine whether or not the previous loop injected a CFE. In case a CFE was injected, a new instance of the virtual platform is created and a handler to the processor is obtained. Next, the processor executes the number of steps indicated by execSteps variable. The execSteps variable indicates how far into the case study the fault injection testing has already progressed. When no CFE was injected, the processor of the current instance of the virtual platform is progressed one step further and the corresponding variables are updated.

Next, the current PC of the processor is read out and the loop variable CFE_Injected is updated. The read PC value is compared to the user specified endAddress value. The endAddress is the PC value that indicates the end of the current fault injection experiment. When this value is reached, all desired CFEs have been injected and the experiment can thus stop. In our case, the endAddress is the return address of the function implementing the target algorithm. In other words, in our case the endAddress is the first PC value of the verification part of the case study.

The next step in the algorithm is to determine whether or not the read out PC falls within the user specified range. When the $\mathrm{PC}$ is not in that range, a new $\mathrm{CFE}$ attempt is started and the execution phase restarts. In case the $\mathrm{PC}$ does fall within the range, its according CFE possibilities are extracted from all possibilities that were created at the start of the algorithm.

Finally, the process analyses whether for the current PC a new CFE must be injected. This is determined by comparing the current wanted possibility index (possIndex) with the number of possibilities for this PC. If a CFE must be injected, it is injected, analysed for its effect on the target program, the according variables are updated and the current instance of the virtual platform is deleted. Then, the execution part of the fault injection restarts.

When no CFE must be injected, a final check is executed. This final check determines whether or not the current PC must be removed from the range of allowed PC values. It does this by checking if the total amount of injected CFEs exceeds the limit provided by the user via the minCFEs option. When the total amount of injected CFEs exceeds the limit, the PC is removed. The minCFEs option is mainly used to break the process out of case studies that have loops with a lot of iterations, also known as hot-loops. In this case, the loop iterations will inject the same CFEs until the minCFEs limit is reached. Once reached, the PC values part of the loop will be removed from the allowed PC range, causing the fault injection experiment to skip them and test the remainder of the target program.

We implemented the fault injection process as a framework to interact with the simulated Cortex-M3 


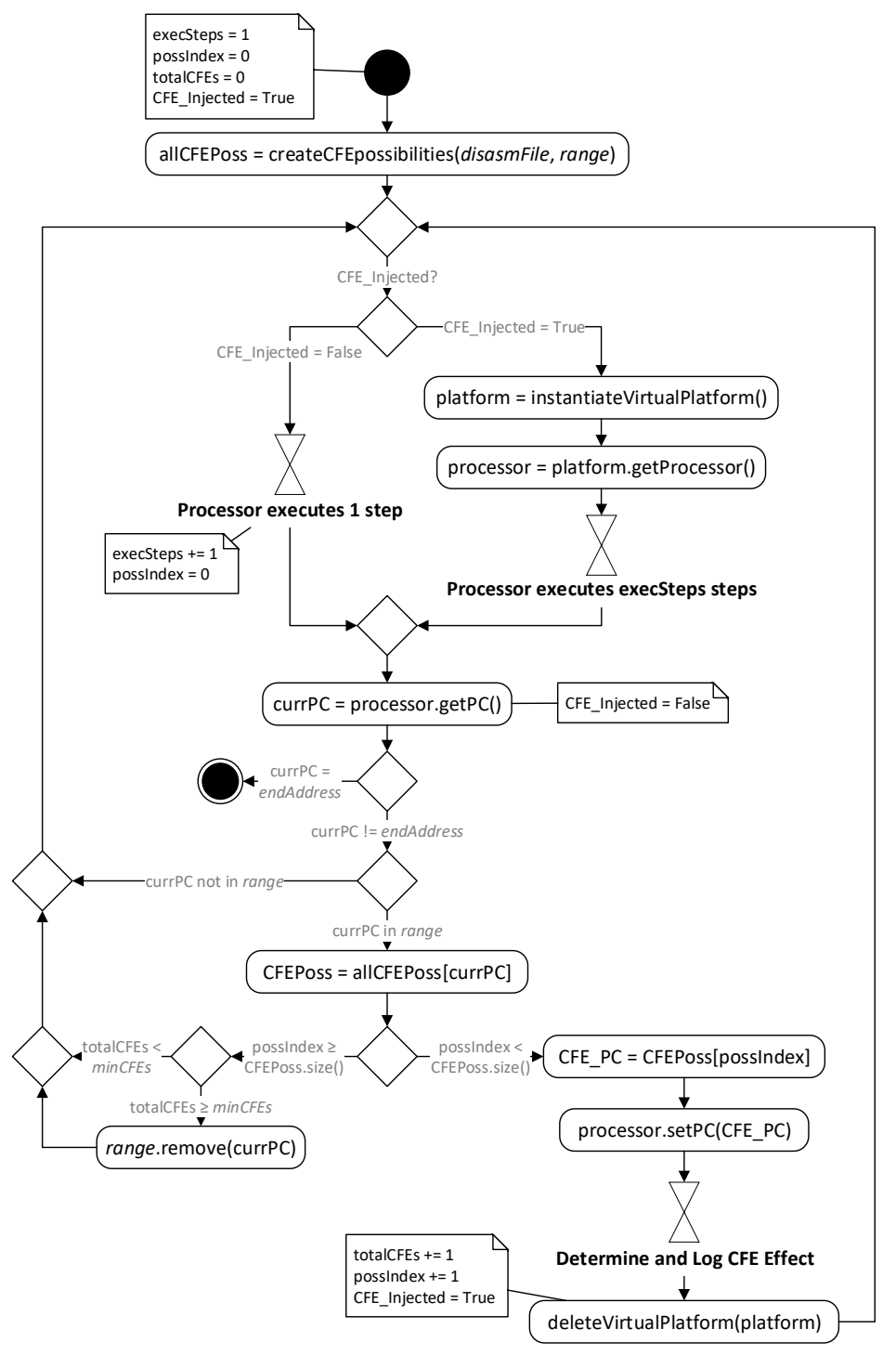

Figure 3: Flowchart showing the used fault injection process. 


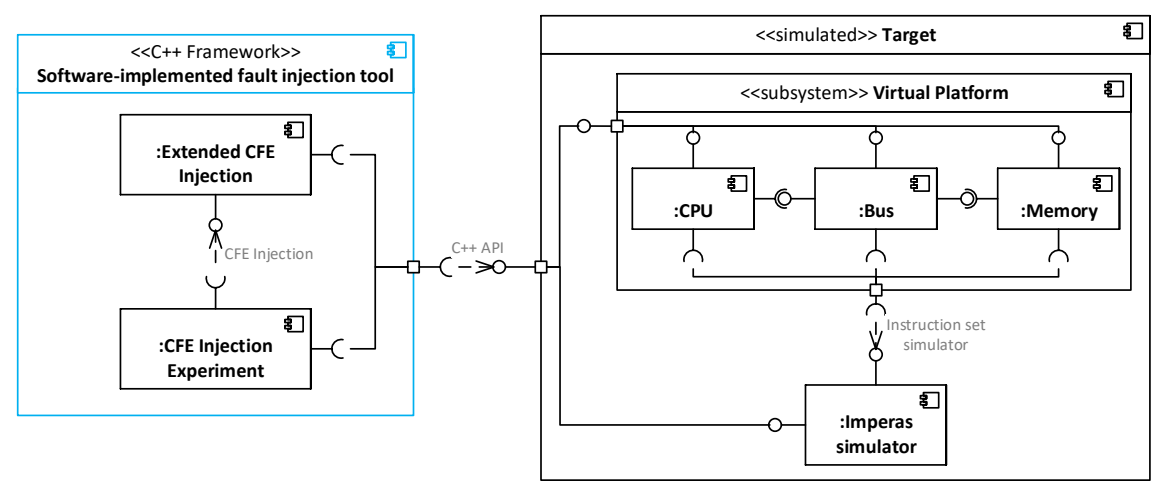

Figure 4: Architecture of the used fault injection tool.

using the Imperas provided C++ API. The built framework interacts with both the simulated CPU as well as with the simulator itself, as shown in Fig. 4. Interaction with the CPU is needed to read and modify the $\mathrm{PC}$ of the simulated ARM Cortex-M3, while interaction with the simulator is needed to advance the CPU and to execute the case study.

\section{Results}

This section represents the results of the performed experiments per defined criterion. In this section, we will only compare the full implementation version with its selective implementation counterpart. Readers interested in the reasons why one technique achieves a higher error detection ratio or imposes a lower overhead than another technique, are referred to our previous work [15, 33, 34].

\subsection{Fault Injection Results}

For each of the selected case studies, we defined five datasets and we subjected each combination of case study, dataset and implemented technique to fault injection experiments. The results are shown in Fig. 5 and Fig. 6 and show the measured ranges of detection ratio and SDC ratio, respectively. In light-blue, the results of the full implementation are shown and in dark-blue, the results of the selective version are shown.

As indicated in Fig. 5, implementing a technique selectively can have a big impact on its error detection ratio. While the full implementations of RSCFC and SIED have an average error detection ratio of $38 \%$ and $65 \%$ respectively, their selective counterparts only achieve an error detection ratio of $2 \%$ and $6 \%$ respectively. This is because these two techniques use local updates for their signatures, i.e. the update for the signatures is implemented as signature $=$ new value. Such updates overwrite any faulty value that might be stored in the signature due to a CFE. This means that S-RSCFC and S-SIED only detect CFEs that jump directly to the exit blocks, causing this low error detection ratio.

Although the YACCA_CMP technique does not use local signature updates, its selective counterpart also seems unable to achieve a high overall error detection ratio. As depicted in the chart, a broad range 


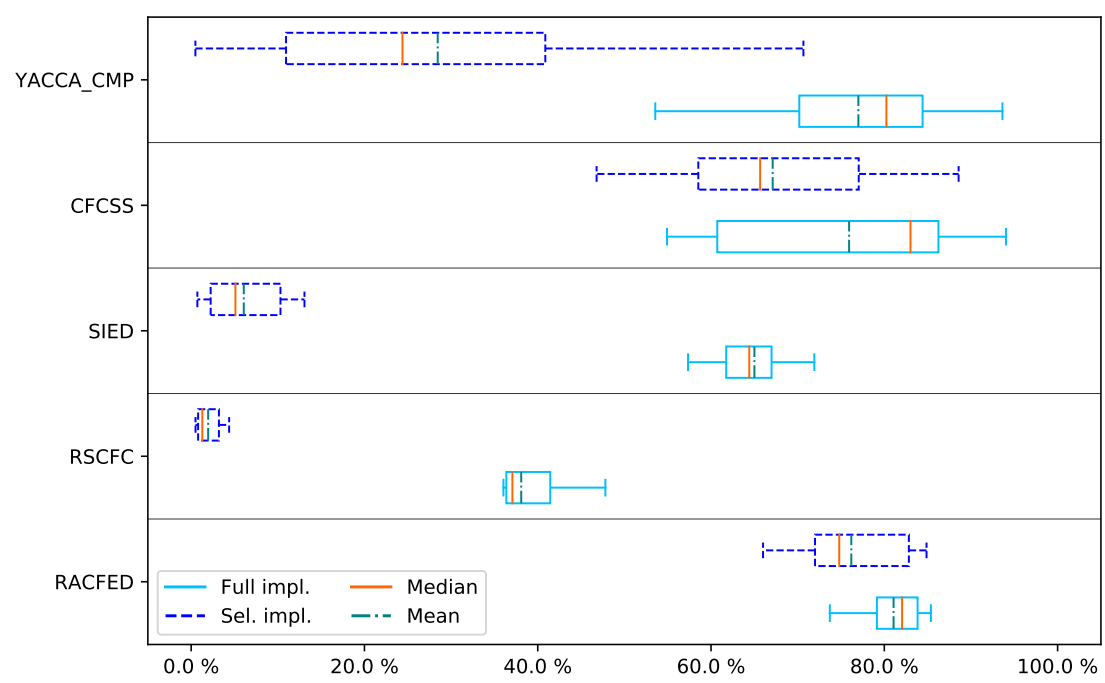

Figure 5: Box plots presenting the error detection ratio measured via the fault injection experiments.

for its error detection ratio was measured with an average of $28 \%$. This low error detection ratio is caused by the bitwise AND operation which is part of the signature update in (S-)YACCA_CMP. For a bitwise AND operation, signature = signature \& bit mask, to be successful, only the bits set in the bit mask need to be set in the signature. Such an operation can thus overwrite a faulty value that might be stored in the signature due to a CFE. The occurrence of such overwriting of faulty information depends from CFE to $\mathrm{CFE}$, explaining the large range of measured error detection ratio for the selective implementation of YACCA_CMP.

Regarding the RACFED and CFCSS techniques, Fig. 5 shows less impact of the selective implementation on the error detection ratio. The average error detection ratio is only a few percentages lower compared to the full implementation and the range of measured values is only slightly larger for the selective version. This small impact is due to the use of gradual updates, i.e. the updates for the signature are implemented as signature = signature operation value. The operation for RACFED is an addition or subtraction with random values, and for CFCSS it is a bitwise EXCLUSIVE OR with a bit mask. Such gradual updates use the current value of the signature to calculate the next value. As shown in previous work, this linked chain of updates leads to a high error detection ratio [33]. The reduction in error detection ratio seen for the selective versions of RACFED and CFCSS is due to the change in memory layout of the code. Because less instructions are inserted, the data showed that more single-bit bit-flip CFEs can now either jump directly to the exit instruction or can exploit weaknesses within the detection techniques.

As indicated in Fig. 6, the lower error detection ratio is coupled to a higher SDC ratio. Since the selective implementation had a major impact on S-RSCFC, S-SIED and S-YACCA_CMP, a significant rise in SDC ratio compared to their full implementation versions was measured. For S-RACFED and S-CFCSS, 


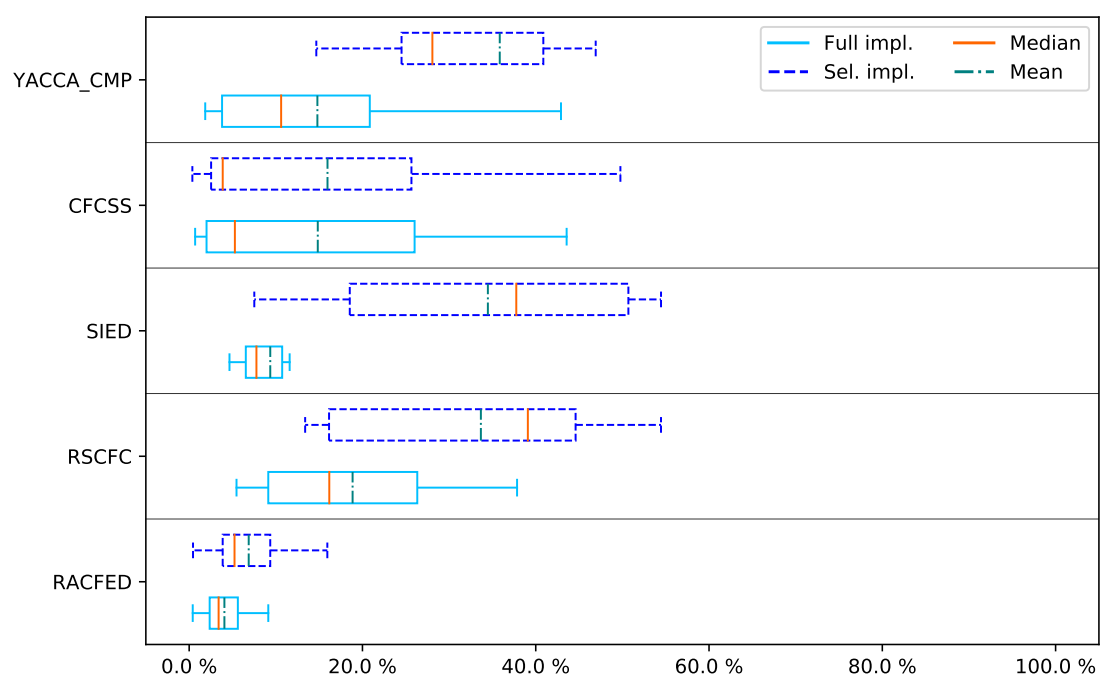

Figure 6: Box plots presenting the silent data corruption ratio measured via the fault injection experiments.

the impact on the SDC ratio is limited and the differences between the full implementation and selective implementations are again due to the difference in the memory layout of the code.

Although little to no difference between the fault injection results of the full implementation and the selective implementation version were expected, the results show that depending on the technique large differences are possible. The fault injection results revealed that our selective implementation approach can only be applied to CFE detection techniques that use gradual updates that depend on more than a few bits to be successful. In all other cases, the proposed approach has a major negative impact on error detection and SDC ratio.

\subsection{Error Detection Cost}

The error detection cost of the techniques, the code size overhead and the execution time overhead, is shown in Fig. 7 and Fig 9 respectively. In light-blue, the overhead of the full implementation shown and in dark-blue, the overhead of the selective version is shown.

As expected, Fig. 7 shows a lower code size overhead for the selective version of each technique. For S-RACFED, S-RSCFC, S-SIED and S-CFCSS, the overall reduction in code size overhead is limited, but it does lower the maximum measured code size overhead. A clear example of this is shown for (S-)SIED. Where the maximum measured value for SIED is a code size overhead of $\times 6.78$, the maximum measured value for S-SIED is $\times 5.33$ which is an outlier and therefore not shown on the chart. For these four techniques, the average code size overhead has reduced with a factor $\times 0.4$. For example, RACFED has an average code size overhead of $\times 3.05$, while S-RACFED has an average code size overhead of $\times 2.65$.

For the YACCA_CMP technique however, a significant reduction in code size overhead is measured. 


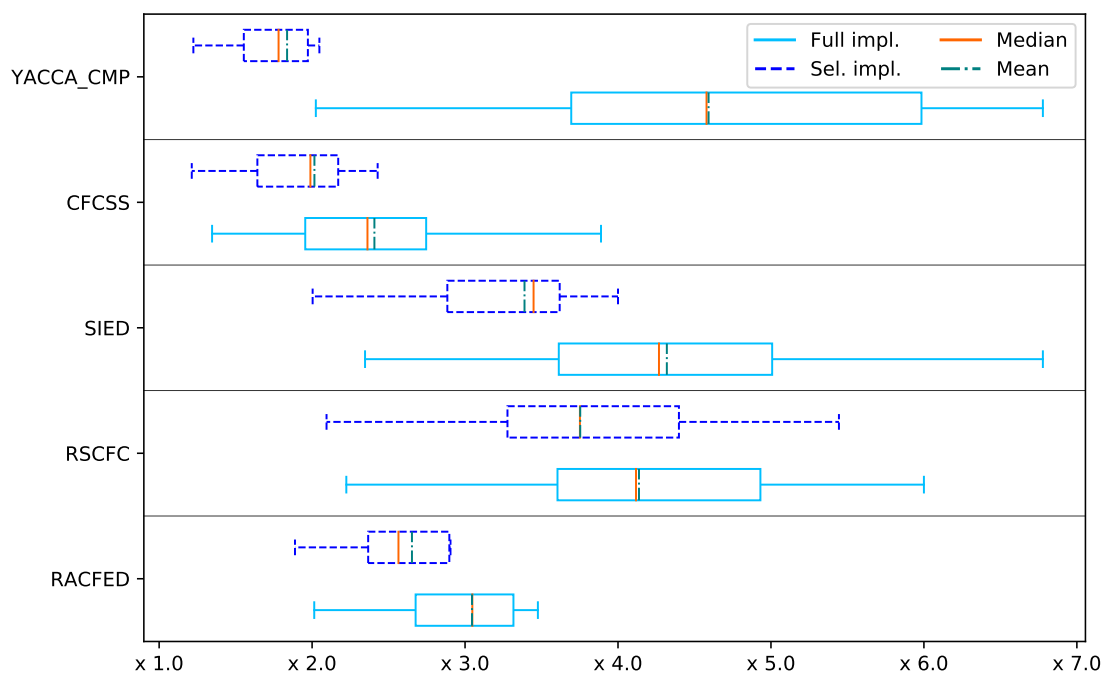

Figure 7: Box plots presenting the code size overhead of the detection techniques.

Where the full version has an average code size overhead of $\times 4.59$ and maximum value of $\times 6.78$, its selective counterpart has an average code size overhead of $\times 1.84$ and a maximum value of $\times 3.00$. Fig. 8 explains the reduction in code size overhead when selectively implementing YACCA_CMP. On the left side, the full implementation of YACCA_CMP is shown. In bold, the instructions added by the technique are shown with their purpose indicated by the curly braces. As shown, most instructions are part of the signature verification. Our proposed selective implementation strategy dictates that signature verification instructions are only inserted in exit basic blocks. Therefore, selectively implementing YACCA_CMP, transforms it to the right side of Fig. 8. Only two instructions are now inserted in each non-exit basic block, hence significantly reducing the imposed code size overhead.

A similar rationale holds for the execution time overhead, as illustrated in Fig 9. The selective implementations have lower maximum values for each technique and the average reduction for S-RACFED, S-RSCFC, S-SIED and S-CFCSS is again $\times 0.4$. For S-YACCA_CMP the reduction is far greater, with an average execution time overhead of $\times 1.23$, while the full implementation has an execution time overhead of $\times 2.77$. This is not surprising, since S-YACCA_CMP only inserts 2 extra instructions for each non-exit basic block as shown in Fig. 8.

Overall, Fig. 7 and Fig 9 show that the proposed selective implementation strategy has a limited impact on the average imposed overhead. It does however limit the maximum measured values for both code size and execution time overhead. 

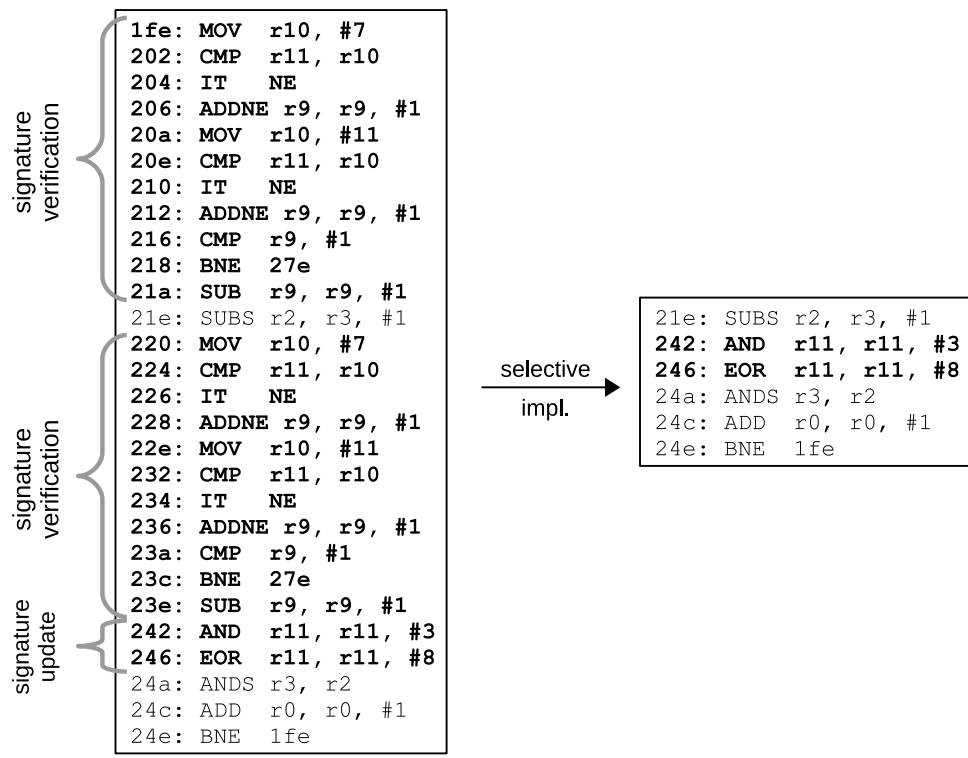

Figure 8: Impact of selectively implementing YACCA_CMP.

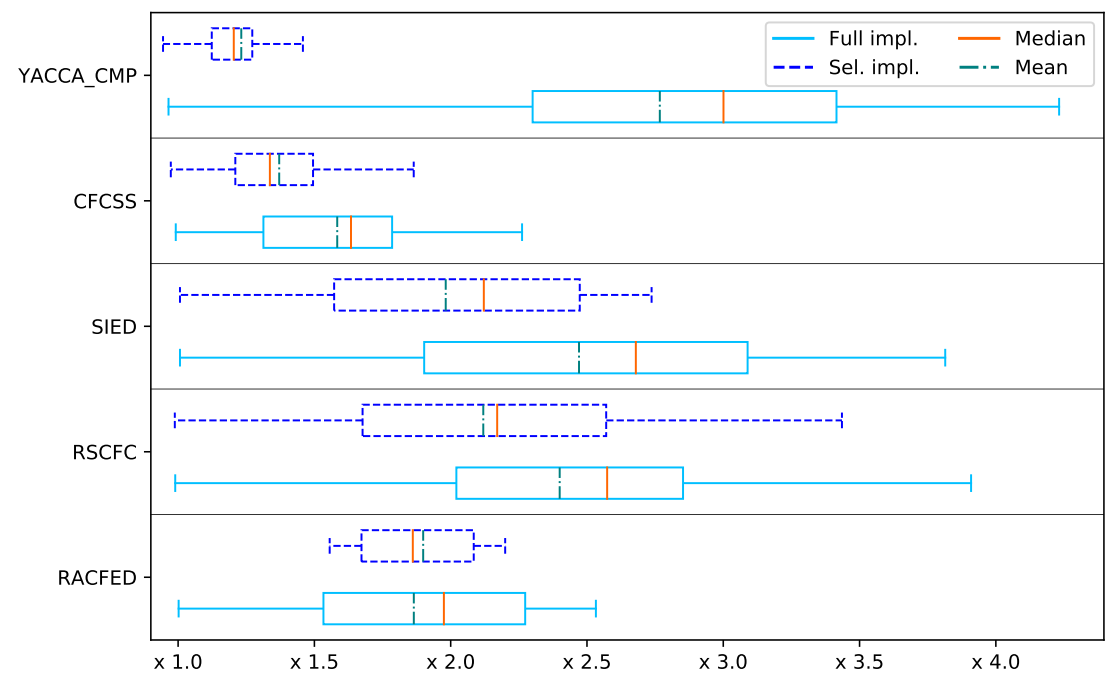

Figure 9: Box plots presenting the execution time overhead of the detection techniques. 


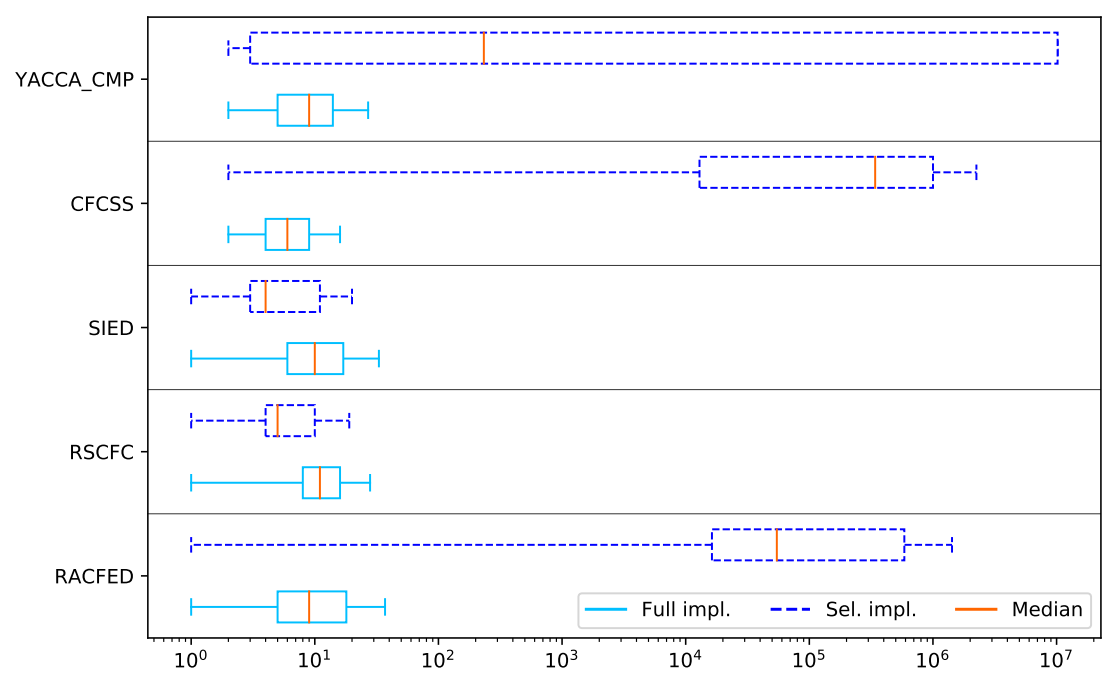

Figure 10: Boxplots showing the extra error detection latency of the selective implementation compared to the full implementation, using a logarithmic $\mathrm{x}$-axis.

\subsection{Error Detection Latency}

While a lower error detection cost is the advantage of selectively implementing a CFE detection technique, its disadvantage is a higher error detection latency. For the performed experiments, we measured the error detection latency as the number of executed instructions between the point of injection and the execution of the error handler. The results are shown in the box plots of Fig. 10, with the error detection latency data for full implementation shown in light-blue and the data for the selective versions depicted in dark-blue. To show the broad range of data as clear as possible, we chose a logarithmic scale on the x-axis.

As illustrated, the error detection latency of the full implementation versions indicate that all detected CFEs are detected within the average basic block length. This is how each technique was designed. For the selective versions, however, we see different results. For S-RSCFC and S-SIED we see a similar error detection latency. While surprising on first sight, it has in fact a logical explanation. The error detection latency is only measured for detected CFEs and as explained in Section 5.1, S-RSCFC and S-SIED only detect CFEs that jump directly to the exit block. Such CFEs are then immediately reported, resulting in a low error detection latency.

For S-RACFED and S-CFCSS the chart depicts the expected results. Some CFEs, e.g. those that jump to an exit basic block, are detected early, but for most CFEs the entire program has to be executed resulting in a high error detection latency. For S-YACCA_CMP the figure shows a larger spread of the data, making it difficult to deduce when a CFE will be detected. As aforementioned, S-YACCA_CMP only detects a CFE if it jump directly to an exit basic block or if the bitwise AND operation does not overwrite a faulty value stored in the signature. The former will result in a low error detection latency and the latter in a high error 
detection latency. Their occurrence, however, is case study dependent.

Overall, these results indicate that the selective implementation strategy is not suited for all types of applications. For example, an application that controls an actuator preferably has a low error detection latency as erroneously controlling an actuator can cause damage or injuries. Applications that are suitable for a selectively implemented error detection ratio are applications that process data and return the result. Since only the result is of importance, a higher detection latency can often be tolerated.

\section{Future Work}

In this paper, we focused on demonstrating that our selective implementation approach can indeed be applied to multiple CFE detection techniques. This does leave the question: how does S-RACFED perform compared to other selective techniques, such as SETA-C and ACS? To answer this question, a similar empirical study, as carried out in this paper, has to be performed. Implementing these selective techniques for the same case studies, executing them on the same case studies and subjecting them to the same fault injection process will reveal which technique achieves the highest error detection ratio, the lowest SDC ratio and the lowest error detection latency.

Furthermore, because our approach and that of SETA-C are quite similar, it might be worthwhile to investigate how these two selective approaches compare to one another. As aforementioned, in SETA-C all signature updates remain and only the comparison instructions are inserted in larger basic blocks. Regarding the signature updates, this strategy is the same is for our selective implementation. For the comparison instructions, other basic blocks are selected compared to our strategy. Therefore, an empirical study might reveal that the SETA-C approach has a lower error detection latency but has less impact on the imposed overhead. To perform this empirical study, we will apply our selective approach to the SETA technique, apply the SETA-C approach to our RACFED technique and re-perform the experiments as described in this paper.

Finally, to further improve our proposed selective implementation approach, the error detection latency has to be reduced. One way to achieve this reduction, is placing comparison instructions in the basic blocks of hot-loops in the target algorithm. As discussed before, these are loops with many iterations. Placing a comparison instruction in such basic blocks enables early error detection, as CFEs can be detected with each iteration of the hot-loop. This does come at the cost of a minor increase in code size overhead and a possibly large increase in execution time overhead, as more instructions need to be executed for each iteration.

\section{Conclusions}

This paper presented a new approach to selectively implement CFE detection techniques. We propose to only insert signature comparisons and the branches to the error handler in the return basic blocks of the 
target algorithm. The advantages of this approach are that it is easy to apply and that it can be applied to most, if not all, existing CFE detection techniques.

We validated this approach by applying it to five CFE detection techniques: our own RACFED technique, the RSCFC technique, the SIED technique, the CFCSS technique and the YACCA_CMP technique. Next, we performed fault injection experiments for eight case studies and measured the error detection ratio, the error detection cost and the error detection latency of both the full implementation and the selective implementation. The results show that only when the technique uses gradual signature updates, of which the result does not depend on a few bits only, our selective implementation strategy has a limited impact on the error detection ratio. This is the case for S-RACFED and S-CFCSS. When local signature updates are used, as is the case for S-RSCFC and S-SIED, very few CFEs are still detected. The same holds for S-YACCA_CMP, for which the result of the signature update depends on a few bits only.

For all techniques, the imposed overhead was reduced when selectively implemented, albeit less than expected. On average, the difference between the full implementation and selective implementation is $\times 0.4$. This reduction in overhead does come at a cost of a high error detection latency. This high latency makes the proposed selective implementation less suited for actuator controlling applications. Its high detection ratio and low execution time overhead do make the strategy very suitable for data processing applications.

\section{References}

[1] R. Baumann, Soft errors in advanced computer systems, IEEE Design Test of Computers 22 (3) (2005) $258-266$.

[2] M. White, Y. Chen, , Tech. Rep. 20100014217, National Aeronotics and Space Administration (NASA) (January 2010). URL https://nepp.nasa.gov/files/16361/08_102_4\%20new\%20del_White.pdf

[3] J. Abella, F. J. Cazorla, E. Quiñones, A. Grasset, S. Yehia, P. Bonnot, D. Gizopoulos, R. Mariani, G. Bernat, Towards improved survivability in safety-critical systems, in: 2011 IEEE 17th International On-Line Testing Symposium, 2011, pp. 240-245. doi:10.1109/IOLTS.2011.5994536.

[4] N. Kanekawa, E. H. Ibe, T. Suga, Y. Uematsu, Dependability in Electronic Systems: Mitigation of Hardware Failures, Soft Errors, and Electro-Magnetic Disturbances, Springer, 2011.

URL https ://www.springer.com/gp/book/9781441967145

[5] B. D. Sierawski, R. A. Reed, M. H. Mendenhall, R. A. Weller, R. D. Schrimpf, S. J. Wen, R. Wong, N. Tam, R. C. Baumann, Effects of scaling on muon-induced soft errors, in: 2011 International Reliability Physics Symposium, 2011, pp. 3C.3.1-3C.3.6. doi:10.1109/IRPS.2011.5784484.

[6] S. Baffreau, S. Bendhia, M. Ramdani, E. Sicard, Characterisation of microcontroller susceptibility to radio frequency interference, in: Proceedings of the Fourth IEEE International Caracas Conference on Devices, Circuits and Systems (Cat. No.02TH8611), 2002, pp. I031-1-I031-5. doi:10.1109/ICCDCS.2002.1004088.

[7] K. Kim, A. A. Iliadis, Critical bit errors in cmos digital inverters due to pulsed electromagnetic interference, in: 2007 International Conference on Electromagnetics in Advanced Applications, 2007, pp. 217-220. doi:10.1109/ICEAA.2007.4387276.

[8] N. A. Estep, J. C. Petrosky, J. W. McClory, Y. Kim, A. J. Terzuoli, Electromagnetic interference and ionizing radiation effects on cmos devices, IEEE Transactions on Plasma Science 40 (6) (2012) 1495-1501. doi:10.1109/TPS.2012.2193600.

[9] S. Jagannathan, Z. Diggins, N. Mahatme, T. D. Loveless, B. L. Bhuva, S. J. Wen, R. Wong, L. W. Massengill, Temperature 
dependence of soft error rate in flip-flop designs, in: 2012 IEEE International Reliability Physics Symposium (IRPS), 2012, pp. SE.2.1-SE.2.6. doi:10.1109/IRPS.2012.6241927.

[10] R. De Keulenaer, Softwarebeveiliging van smartcards tegen laseraanvallen, Master's thesis, Universiteit Gent (2013).

[11] A. Tang, S. Sethumadhavan, S. Stolfo, CLKSCREW: Exposing the perils of security-oblivious energy management, in: 26th USENIX Security Symposium (USENIX Security 17), USENIX Association, Vancouver, BC, 2017, pp. 1057-1074.

[12] E. H. Ibe, S. Yoshimoto, M. Yoshimoto, H. Kawaguchi, K. Kobayashi, J. Furuta, Y. Mitsuyama, M. Hashimoto, T. Onoye, H. Kanbara, H. Ochi, K. Wakabayashi, H. Onodera, M. Sugihara, VLSI Design and Test for Systems Dependability, Springer Japan, 2019, Ch. Radiation-Induced Soft Errors. doi:10.1007/978-4-431-56594-9_3.

[13] A. Li, B. Hong, Software implemented transient fault detection in space computer, Aerospace Science and Technology 11 (2) (2007) 245 - 252. doi:10.1016/j.ast.2006.06.006.

[14] B. Nicolescu, Y. Savaria, R. Velazco, SIED: software implemented error detection, in: Proceedings 18th IEEE Symposium on Defect and Fault Tolerance in VLSI Systems, 2003, pp. 589-596.

[15] J. Vankeirsbilck, N. Penneman, H. Hallez, J. Boydens, Random additive control flow error detection, in: B. Gallina, A. Skavhaug, F. Bitsch (Eds.), Computer Safety, Reliability, and Security, Springer International Publishing, Cham, 2018, pp. 220-234.

[16] E. Chielle, G. S. Rodrigues, F. L. Kastensmidt, S. Cuenca-Asensi, L. A. Tambara, P. Rech, H. Quinn, S-SETA: Selective software-only error-detection technique using assertions, IEEE Transactions on Nuclear Science 62 (6) (2015) 3088-3095. doi:10.1109/TNS.2015.2484842.

[17] D. S. Khudia, S. Mahlke, Low cost control flow protection using abstract control signatures, SIGPLAN Not. 48 (5) (2013) 3-12. doi:10.1145/2499369.2465568.

[18] N. Oh, P. P. Shirvani, E. J. McCluskey, Control-flow checking by software signatures, IEEE Transactions on Reliability 51 (1) (2002) 111-122.

[19] O. Goloubeva, M. Rebaudengo, M. Sonza Reorda, M. Violante, Soft-error detection using control flow assertions, in: Proceedings of the 18th IEEE International Symposium on Defect and Fault Tolerance in VLSI Systems, DFT '03, IEEE Computer Society, Washington, DC, USA, 2003, pp. 581-588.

[20] Z. Alkhalifa, V. S. S. Nair, N. Krishnamurthy, J. A. Abraham, Design and evaluation of system-level checks for on-line control flow error detection, IEEE Trans. Parallel Distrib. Syst. 10 (6) (1999) 627-641. doi:10.1109/71.774911.

[21] S. A. Asghari, A. Abdi, H. Taheri, H. Pedram, S. Pourmozaffari, SEDSR: Soft error detection using software redundancy, Journal of Software Engineering and Applications 5 (9) (2012) 664-670. doi:10.4236/jsea.2012.59078.

[22] S. A. Asghari, H. Taheri, H. Pedram, O. Kaynak, Software-based control flow checking against transient faults in industrial environments, IEEE Transactions on Industrial Informatics 10 (1) (2014) 481-490. doi:10.1109/TII.2013.2248373.

[23] X. Guang, Z. Zhang, Linear Network Error Correction Coding, 1st Edition, SpringerBriefs in Computer Science, SpringerVerlag New York, 2014. doi:10.1007/978-1-4939-0588-1.

[24] R. Zhu, Y. Ma, Information Engineering and Applications, 1st Edition, Vol. 154 of Lecture Notes in Electrical Engineering, Spinger-Verlag London, 2012.

[25] H. König, Protocol Engineering, Springer-Verlag Berlin Heidelberg, 2012. doi:10.1007/978-3-642-29145-6.

[26] R. Dorf, Systems, Controls, Embedded Systems, Energy, and Machines, The Electrical Engineering Handbook, CRC Press, 2016.

[27] S. C. Satapathy, V. Bhateja, A. Joshi, Proceedings of the International Conference on Data Engineering and Communication Technology, 1st Edition, Vol. 469 of Advances in Intelligent Systems and Computing, Springer Singapore, 2017.

[28] W. Fischer, Digital Television: a Practical Guide for Engineers, Springer-Verlag Berlin Heidelberg, 2004. doi:10.1007/9783-662-05429-1.

[29] R. C. Gonzalez, R. E. Woods, Digital Image Processing, Pearson, 2017. 
[30] M. R. Guthaus, J. S. Ringenberg, D. Ernst, T. M. Austin, T. Mudge, R. B. Brown, Mibench: A free, commercially representative embedded benchmark suite, in: Proceedings of the Fourth Annual IEEE International Workshop on Workload Characterization. WWC-4 (Cat. No.01EX538), 2001, pp. 3-14. doi:10.1109/WWC.2001.990739.

[31] J. Vankeirsbilck, H. Hallez, J. Boydens, Automatic implementation of control flow error detection techniques, in: accepted at IASED International Conference on Wireless Networks and Embedded Systems (ICWNES), 2019.

[32] Imperas, Revolutionizing embedded software development, Online (2018). URL http://www.imperas.com/

[33] J. Vankeirsbilck, N. Penneman, H. Hallez, J. Boydens, Random additive signature monitoring for control flow error detection, IEEE Transactions on Reliability 66 (4) (2017) 1178-1192. doi:10.1109/TR.2017.2754548.

[34] J. Vankeirsbilck, Advancing control flow error detection techniques for embedded software using automated implementation and fault injection, Ph.D. thesis, KU Leuven (January 2020). 